\title{
Limnological characterization of floodplain lakes in Mamirauá Sustainable Development Reserve, Central Amazon (Amazonas State, Brazil)
}

\author{
Caracterização limnológica dos lagos da planície de inundação \\ na Reserva de Desenvolvimento Sustentável Mamirauá, \\ Amazônia Central (Estado do Amazonas, Brasil)
}

Adriana Gomes Affonso ${ }^{1}$, Helder Lima de Queiroz ${ }^{2}$ and Evlyn Márcia Leão de Moraes Novo ${ }^{1}$

${ }^{1}$ Divisão de Sensoriamento Remoto, Instituto Nacional de Pesquisas Espaciais, Av. dos Astronautas, 1758, Jardim da Granja, CEP 12227-010, São José dos Campos, SP, Brazil e-mails: affonso@dsr.inpe.br, evlyn@dsr.inpe.br

${ }^{2}$ Instituto de Desenvolvimento Sustentável Mamirauá, Estrada do Bexiga, 2584, CEP 69470-000, Fonte Boa, Tefé, AM, Brazil e-mail: helder@mamiraua.org.br

\begin{abstract}
Aim: This paper examines the spatial and temporal variation of limnological characteristics of floodplain lakes in the Solimóes and Japurá confluence, an undisturbed region - the Mamirauá Sustainable Development Reserve (MSDR); Methods: We analyzed surface temperature, conductivity, $\mathrm{pH}$, dissolved oxygen, turbidity and transparency, and surface water samples were collected for determination of suspended inorganic and organic matter, chlorophyll- $a$, pheophytin, total nitrogen, total phosphorus, organic and inorganic carbon, in two phases of the hydrograph stage, 2009 high water phase, 2008, 2009 and 2010 low water phases; Results: The results showed that the studied water bodies have high variability in all measured variables: a) between hydrograph phases; b) among main rivers; and c) between opposite margins of Japurá River; Conclusions: This shows the remarkable influence of the flood pulse and the primary water source on the limnology of this system. The monitoring of physical and chemical limnological variables in Mamirauá will serve as future reference for comparison with disturbed areas, such as the Lower Amazon, and as a baseline for modeling the effects of climate change and anthropogenic influences on Amazon aquatic ecosystem.
\end{abstract}

Keywords: limnology, undisturbed floodplain lakes, Central Amazon, Mamirauá Reserve.

Resumo: Objetivo: Esse trabalho investiga a variação temporal e espacial de algumas características limnológicas dos lagos da planície de inundação da regiáo de confluência dos Rios Japurá e Solimões, onde está localizada a Reserva de Desenvolvimento Sustentável Mamirauá (RDSM); Métodos: Analisaram-se temperatura, condutividade, $\mathrm{pH}$, oxigênio dissolvido, turbidez e transparência da água em três profundidades (superfície, Secchi e no limite da zona fótica) e coletadas amostras de água na superfície para a determinação de material orgânico e inorgânico em suspensão, clorofila- $a$, feofitina, nitrogênio e fósforo total, carbono orgânico e inorgânico dissolvido, em duas fases da hidrógrafa, água alta em 2009, e água baixa em 2008, 2009 e 2010; Resultados: Os resultados mostraram que as variáveis medidas possuem uma alta variabilidade nos corpos d'água na região de estudo: a) entre as fases da hidrógrafas; b) entre os rios principais; e c) entre as margens opostas do Rio Japurá, mostrando a importância do pulso de inundaçáa na variação dos parâmetros físicos e químicos e ainda a em relação ao rio principal de alimentação; Conclusóes: $\mathrm{O}$ monitoramento dos parâmetros físicos e químicos em Mamirauá servirá como futura referência para comparação com outras regiōes menos preservadas, como o Baixo Amazonas, e ainda como linha de base para modelos sobre efeitos das mudanças climáticas e influências antropogênicas no ecossistema aquático Amazônico.

Palavras-chave: limnologia, planície de inundação não perturbada, Amazônia Central, Reserva Mamirauá. 


\section{Introduction}

The Amazon basin is the largest drainage basin in the world with an area of approximately $6,869,000 \mathrm{~km}^{2}$ (Neill et al., 2006). Due to its geological and geomorphological evolution, the basin has a vast floodplain (várzea) which is formed by a complex system of rivers, channels, lakes and islands that are in constant change due to sedimentation and particulate matter transport. This floodplain is seasonally flooded by different water types that vary widely, depending on its origin, soil type and climatic conditions. Sioli (1984) classified the Amazonian waters into three major groups based on suspended and dissolved matter and $\mathrm{pH}$, whereas, black waters are those with high humic contents, low suspended matter and $\mathrm{pH}$ between 3.8 to 4.9; white waters has high concentration of dissolved and suspended matter and $\mathrm{pH}$ between 6.2-7.2, and clear waters with low turbidity and low content of suspended matter and humic content and $\mathrm{pH}$ ranging from 4.5 to 7.8 . Furthermore, the periodic flooding changes the proportion of suspended and dissolved components in water by altering its physical-chemical conditions (Melack and Forsberg, 2001) consequently, affects the ecosystem where these waters circulate (Forsberg et al., 1988) and also the ecology and life cycle dynamic of local species (Saint-Paul et al., 2000).

The flood pulse is a key factor in the ecological processes of the várzea, which transforms periodically terrestrial environments into aquatic environments (Junk et al., 1997). This dynamic provides a variety of habitats for many animal and plant species (Junk and Da Silva, 1997), provides food sources, nesting and refuge for many fish species (SanchezBotero and Araujo-Lima, 2001). The floodplain is also important for due to its high biodiversity (Junk et al., 2000), high primary productivity of wetland forests (Parolin et al., 2004), aquatic macrophytes (Piedade et al., 1984; Silva et al., 2010), and also provides the main energy source for Amazonian aquatic food chain (Forsberg et al., 1993; Arraut et al., 2010).

The water characteristics and its dynamics are crucial to understand the processes and dynamics that occur between terrestrial and aquatic ecosystems. Several studies have been conducted to assess the spatial and temporal variations of physical and chemical properties of Amazonian waters (Melack and Fisher, 1990; Moreira-Turcq et al., 2003; Aufdenkampe, et al., 2007; McClain and Naiman, 2008; Almeida and Melo, 2009).
However the dynamic nature of the aquatic systems makes it difficult to distinguish between natural variability and anthropogenic influences (Chipps et al., 2006), and even more difficult in the Amazon floodplain lakes, where the flood pulse controls the ecosystem dynamics. Thus the conversion of forest into pasture and agricultural areas in floodplains, especially in eastern Amazon, might affect the water characteristics (Melack and Forsberg, 2001; Tundisi et al., 2002). Therefore, information about water physical and chemical characteristics of undisturbed regions provides a baseline upon which anthropogenic effects on aquatic system may be assessed.

Following this approach this paper examines the spatial and temporal variation of some limnological characteristics of floodplain lakes in the area of confluence of Solimões and Japurá rivers in Central Amazon, where the Mamirauá Sustainable Development Reserve is located. In spite of the fact that it cannot be considered a completely undisturbed várzea due to the human population living there, in this paper, one assumes that it is an almost undisturbed várzea.

\section{Material and Methods}

The study area is situated in western Amazon, near Tefé municipality $(570 \mathrm{~km}$ distant from Manaus), in Amazonas State. It is located in Mamirauá Sustainable Development Reserve (MSDR), which was established in 1990 by state government, and it has an area of 1,124.000 ha approximately (Figure 1). The region is formed by "várzea", a floodplain inundated by sedimentrich whitewater rivers of the Amazon River with a complex mosaic of seasonally inundated forests, lakes and channels, with an annual water level variation of $12 \mathrm{~m}$ (Ayres, 1993; Henderson, 1999; Queiroz, 2005; Queiroz, 2007; Ramalho et al., 2009).

According to the hydrological monitoring data system of the Eastern Amazon, in the hydrological period of 2008/2009 the flooding reached the highest water level of the last 107 years (CPRM, 2009) at Negro/Solimóes River system, near Manaus municipality.

In Mamirauá study area the hydrological period of 2008/2009 corresponded to the second highest record (38.33 $\mathrm{m}$ above sea level) in the historical data (from 1990 to 2010). The highest one occurred in 1999, when in the water reached $38.55 \mathrm{~m}$ above sea level (m.a.s.l.) (Ramalho et al., 2009). Nonetheless, during the drought of 2010, water level reached the 


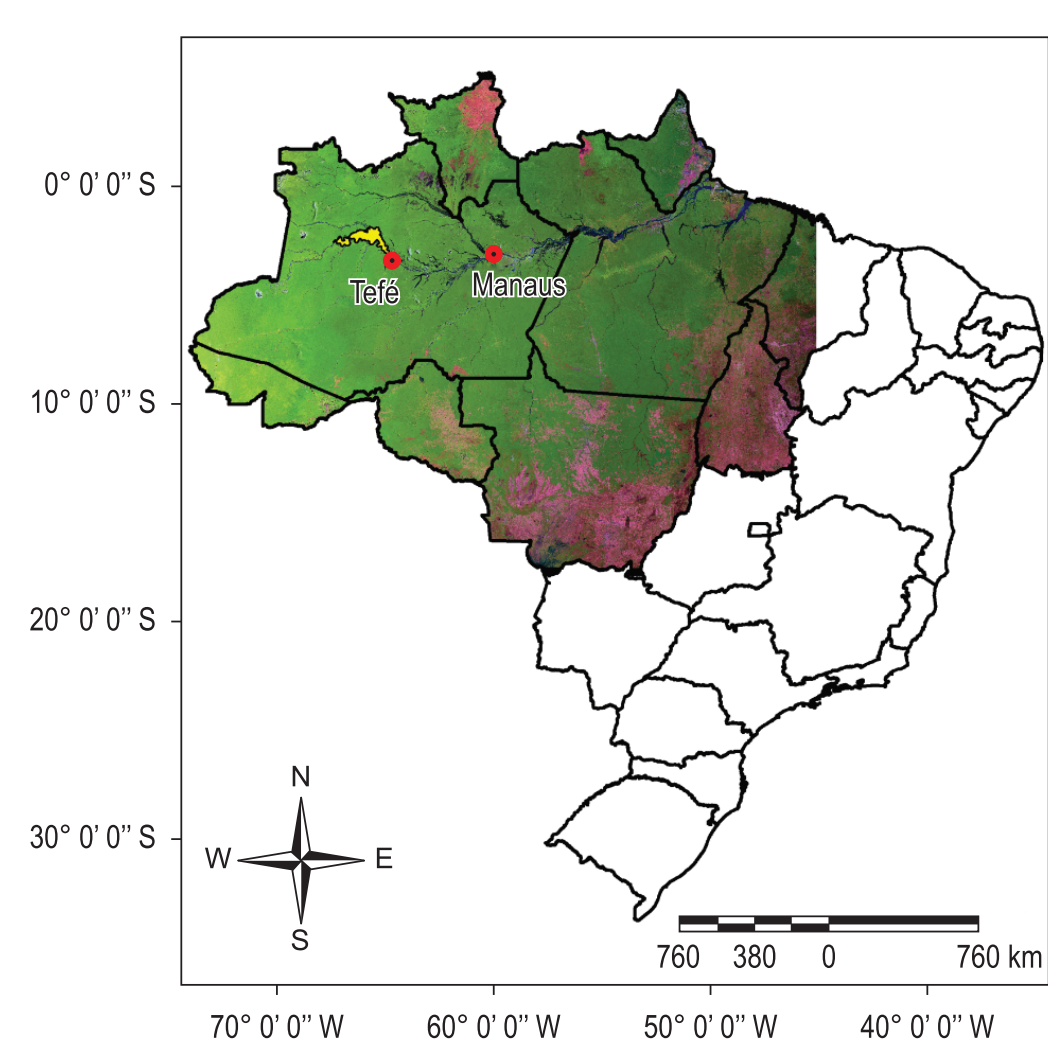

(a)

$70^{\circ} 0^{\prime} 0^{\prime \prime} \mathrm{W} \quad 60^{\circ} 0^{\prime} 0^{\prime \prime} \mathrm{W} \quad 50^{\circ} 0^{\prime} 0^{\prime \prime} \mathrm{W} \quad 40^{\circ} 0^{\prime} \mathrm{O}^{\prime \prime} \mathrm{W}$
- Cities

Mamirauá sustainable development reserve

(b)

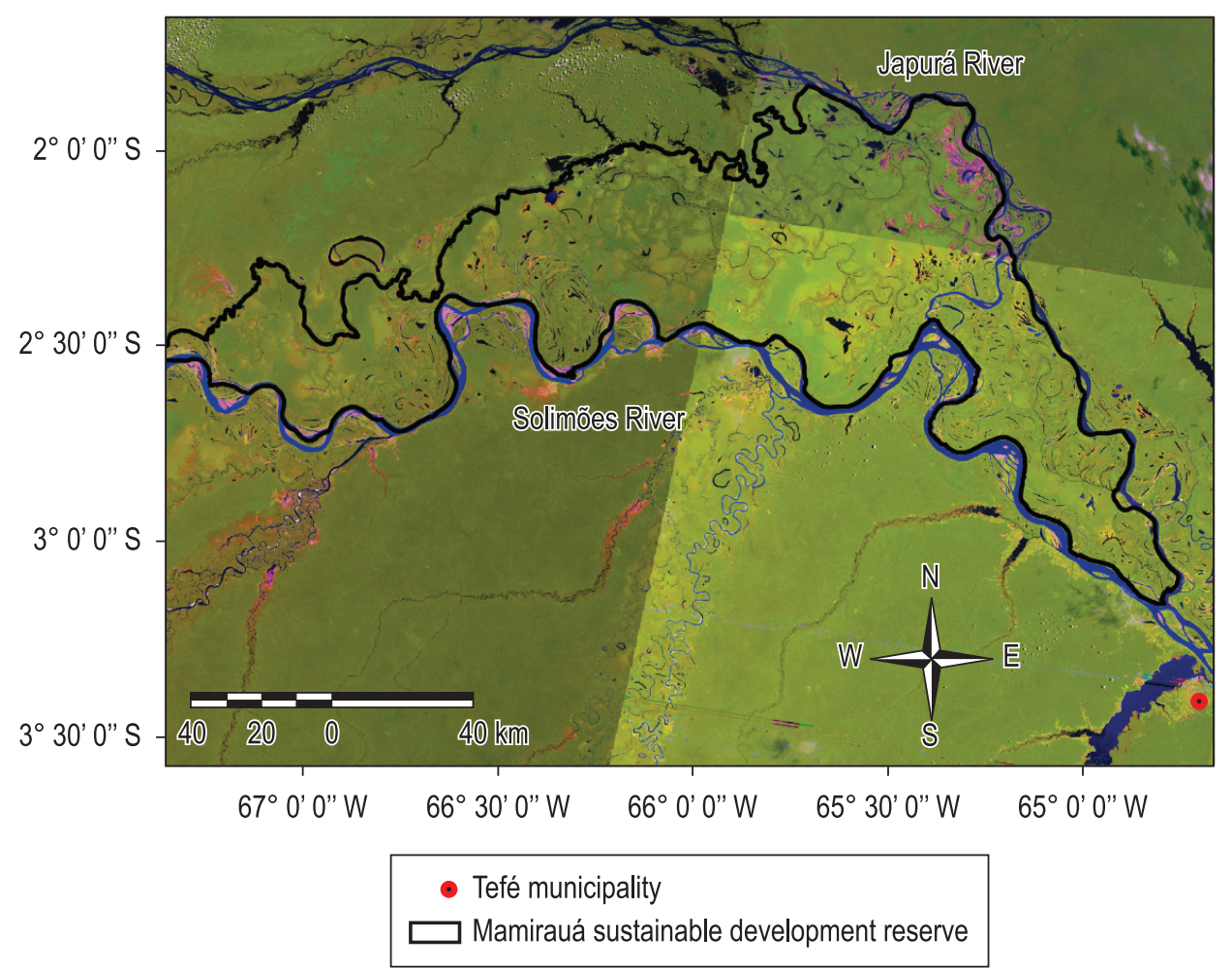

Figure 1. Study area. a) Location of study area in Brazil. Tefé and Manaus municipality and Mamirauá Sustainable Development Reserve (MSDR) are shown; b) Complete Landsat TM image view of MSDR. Japurá and Solimóes Rivers are shown. 
lowest level for the last 108 years, lower than 1963 or 2005, when water during the droughts reached 13.64 and 14.75 m.a.s.l., respectively, at Manaus. In Mamirauá study area, however, the 2010 low water phase was considered as one of the driest in in the historical data, with the minimum water level of $24.4 \mathrm{~m}$. The driest ever for the time series available for Mamirauá occurred in 1995 when the water level reached 21.71 m.a.s.l., followed by the 1991, 1992 and 2005 droughts (with 23.45, 23.41 and 24.09 m.a.s.l., respectively) (Ramalho et al., 2009). Thus this paper presents limnological data about two historical, extreme and opposite events, and also presents data of regular events.

Field samples were distributed among floodplain lakes, located on the right and left margins of Japurá River, and also in the main rivers (Japurá and Solimóes Rivers) in 2008 (low water phase), 2009 (high and low water phase) and 2010 (low water phase) (Figure 2 and 3). Water temperature $\left({ }^{\circ} \mathrm{C}\right)$, dissolved oxygen (mg. $\left.\mathrm{L}^{-1}\right)$, turbidity (NTU), electrical conductivity $\left(\mu S . \mathrm{cm}^{-1}\right)$ and $\mathrm{pH}$ were measured on the surface using an YSI multiparameter probe. Surface water samples were also collected for determination of the following parameters in the laboratory: suspended inorganic
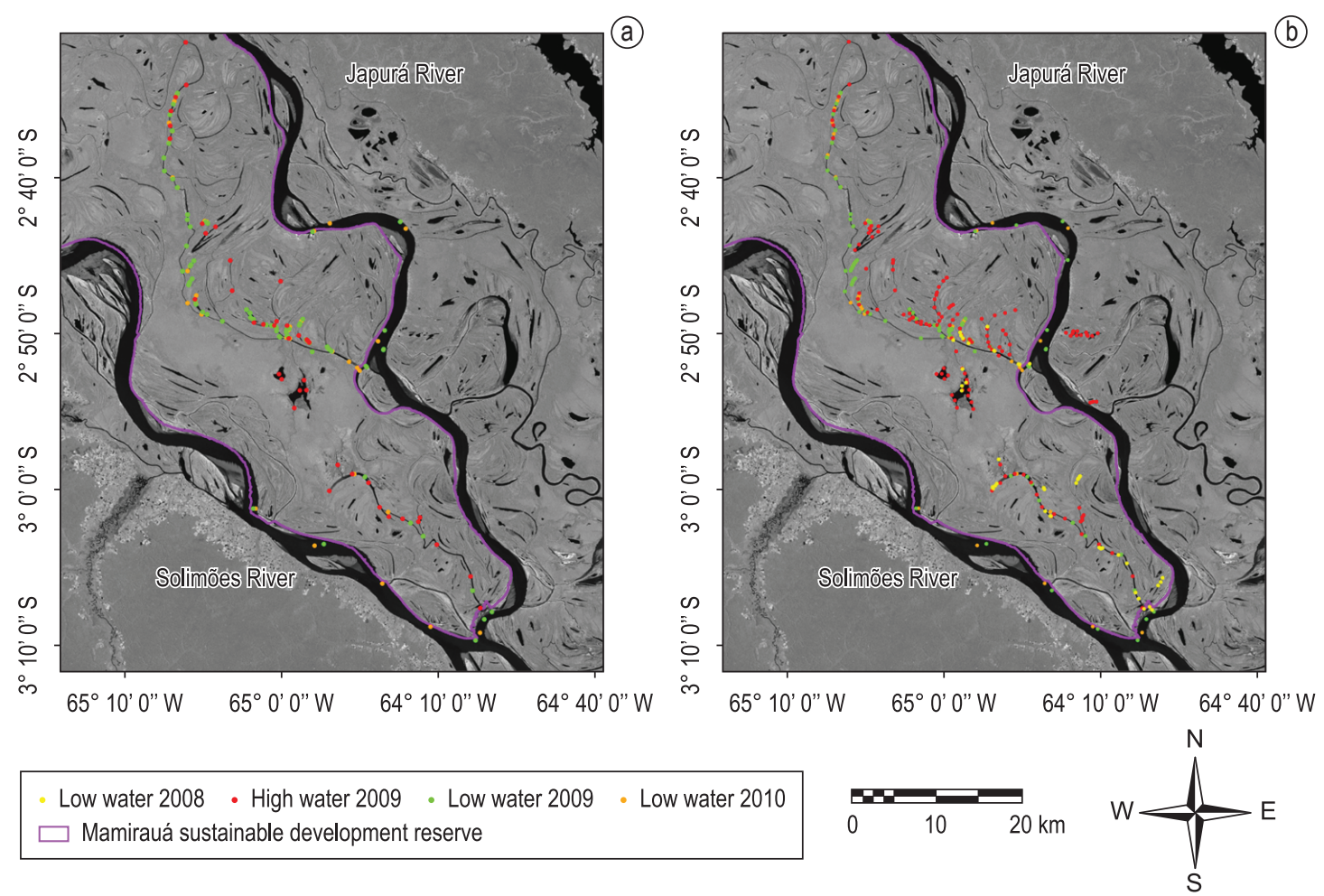

Figure 2. Complete (a) and incomplete (b) sampling stations at study area, whereas, 2008 low water sampling points are show in yellow dots, 2009 high water in red, 2009 low water in green and 2010 low water in orange. Japurá and Solimóes river are shown. and organic matter $\left(\mathrm{mg} . \mathrm{L}^{-1}\right)$; chlorophyll- $a\left(\mu \mathrm{g} . \mathrm{L}^{-1}\right)$, pheophytin $\left(\mu \mathrm{g} . \mathrm{L}^{-1}\right)$, total nitrogen $\left(\mathrm{mg} . \mathrm{L}^{-1}\right)$, total phosphorus $\left(\mu \mathrm{g} . \mathrm{L}^{-1}\right)$, organic and inorganic carbon (mg. $\left.\mathrm{L}^{-1}\right)$.

For chlorophyll-a, pheophytin and suspended inorganic and organic matter, water samples were filtered (Whatmann $\mathrm{GF} / \mathrm{C}$ fiberglass filter $(0.5-0.7 \mathrm{~mm}))$ and stored in silica gel at $0{ }^{\circ} \mathrm{C}$ until further analysis in laboratory according to Nush (1980) and Wetzel and Likens (1991) methodology, respectively. Total phosphorus and nitrogen were determined through Valderrama (1981) and Golterman et al. (1978), in that order, and organic and inorganic carbon according to Eaton et al. (1995).

The number of samples varied in different hydrograph phases (Table 1) as the open water extent changed from one phase to other, limiting the access to some lakes and regions. For the lakes located on the left margin of Japurá River, samples were taken only during 2009 high water phase, because of the limited access during low water phase campaigns.

There were two types of sampling stations: 1) Complete sampling: both surface water samples
Low water 2008 • High water 2009 - Low water 2009 Mamirauá sustainable development reserve 
and YSI measurements, and 2) Incomplete sampling: only YSI probe measurements.

Limnological data were submitted to a twosample non-parametric test Kolmogorov-Smirnov $(\mathrm{p}<0.01)$ (Siegel, 1975) to investigate if there were statistically significant differences in water properties among samples collected in: i) different phases of the flood pulse (flood and drought), ii) different margins of the Japura River, iii) Solimóes and Japurá Rivers, and also, iv) distinct drought events.

The two-sample Kolmogorov-Smirnov test uses the maximal distance (Dmax) between cumulative frequency distributions of each two samples, and, if the Dmax is greater than the critical value (Dcrit),

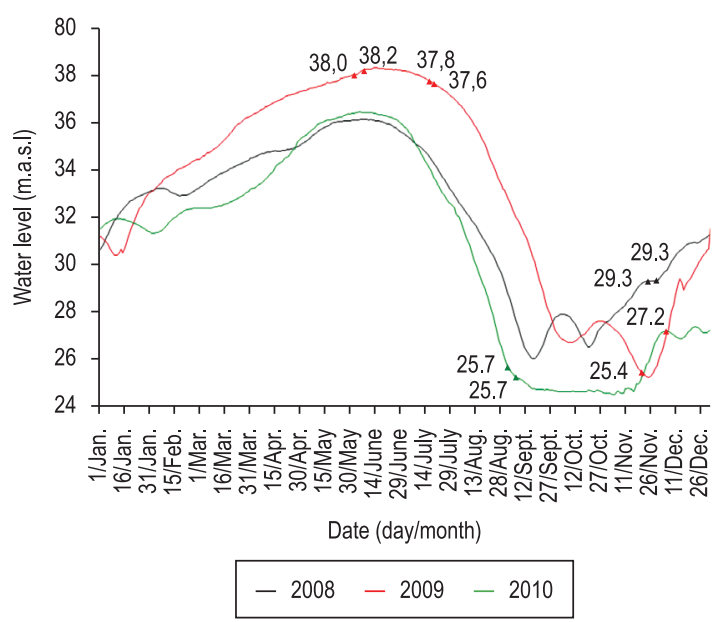

Figure 3. Water level variation (above sea level) during 2008, 2009 and 2010 field campaigns and the respective water level in the beginning and end of each field mission. which is obtained from a table, than these samples come from different distribution.

\section{Results}

In general, the magnitude of all variables was higher in drought (or low water phase - LWP) than in rainy season (or high water phase - HWP), except for water transparency (Tables 2 to 5). The lakes located on the right margin of Japurá River had higher transparency, $\mathrm{pH}$ and electrical conductivity values than those on the opposite margin, during high water phase. The left margin lakes were warmer, more turbid and with higher oxygen concentration in the same season. The two-sample KolmogorovSmirnov tests (K-S test) showed that the differences reported were statistically significant, as described in Tables 6 to 9.

Japurá River had lower values than Solimóes River in all variables, except for dissolved oxygen and chlorophyll-a, during LWP. KolmogorovSmirnov (K-S test) tests showed that turbidity (in 2009) and dissolved oxygen (in 2010) values displayed differences statistically significant between those rivers, during low water phases. When comparing drought events $(2009 \times 2010)$, conductivity, temperature, turbidity and $\mathrm{pH}$ measured at Japurá river displayed statistically significant differences, and for the Solimóes River, only turbidity and dissolved oxygen were statistically different (Tables 6 to 9).

When comparing the 2008 to the 2009 and 2010 drought events, the electrical conductivity, $\mathrm{pH}$ and turbidity distributions were statistically different (Tables 6 to 9). Dissolved oxygen was

Table 1. Number of sampling points collected per sampling type in different hydrograph phases/year.

\begin{tabular}{ccccc}
\hline Hydrograph phase & 2008 low water & 2009 high water & 2009 low water & 2010 low water \\
\hline Sampling date & $24 / 11$ to $29 / 11$ & $20 / 05$ to $11 / 06$ & $11 / 11$ to $10 / 12$ & $29 / 08$ to $07 / 09$ \\
Complete & NA & 41 & 74 & 45 \\
Incomplete & 50 & 136 & 97 & 64 \\
\hline
\end{tabular}

NA: Not applicable. It was not collected water surface samples during this field work.

Table 2. Mean, minimum (Min), maximum (Max), coefficient of variation (Coeff.var.) and number of samples (N) of variables measured during 2008 low water field mission.

\begin{tabular}{|c|c|c|c|c|c|}
\hline Variables & Mean & Min & Max & Coeff.Var. & $\mathrm{N}$ \\
\hline Transparency (m) & 0.56 & 0.15 & 1.20 & 54.94 & 50 \\
\hline Temperature $\left({ }^{\circ} \mathrm{C}\right)$ & 31.31 & 29.24 & 33.60 & 4.59 & 50 \\
\hline $\mathrm{pH}$ & 6.99 & 6.60 & 8.17 & 4.38 & 50 \\
\hline $\mathrm{EC}\left(\mu \mathrm{S} . \mathrm{cm}^{-1}\right)$ & 156.21 & 61.00 & 300.90 & 38.40 & 50 \\
\hline Turbidity (NTU) & 23.82 & 3.92 & 158.90 & 125.49 & 50 \\
\hline DO (mg. L-1) & 4.23 & 0.77 & 10.32 & 60.33 & 50 \\
\hline
\end{tabular}

EC: Electrical conductivity; DO: dissolved oxygen. 
statistically different when comparing two different drought events: 2008 (a mild drought episode) and 2010 (an extreme drought event).

The 2009 and 2010 low water phase events were statistically different for water transparency, dissolved oxygen, chlorophyll- $a$, pheophytin, nitrogen, phosphorus, and inorganic and organic dissolved carbon (Tables 6 to 9).

Mean water transparency (Secchi depth) were higher during HWP (1.58 $\mathrm{m}$ than in drought events when ranged from 0.32 to $0.44 \mathrm{~m}$, with a maximum value of $1.2 \mathrm{~m}$ in 2008 and the minimum transparency of $0.05 \mathrm{~m}$ in 2009 (Tables 2 to 5). Right margin lakes were more transparent than left margin lakes during HWP and also than main rivers during LWP. Solimôes and Japurá Rivers had the lowest mean values, with less than $0.2 \mathrm{~m}$ of water transparency in both dry periods.

The lowest water temperature was recorded during HWP $\left(26.5^{\circ} \mathrm{C}\right)$ and the highest occurred in 2010 drought $\left(34.7^{\circ} \mathrm{C}\right)$. Mean values during LWP varied from 31.22 (2009) to 31.33 (2010). Mean temperature of right margin lakes $\left(27.08^{\circ} \mathrm{C}\right)$ were lower than left margin lakes $\left(28.04^{\circ} \mathrm{C}\right)$ during HWP, but higher $\left(31.55^{\circ} \mathrm{C}\right)$ than main rivers $\left(30.12^{\circ} \mathrm{C}\right.$ ) during LWP (Tables 2 to 5$)$.

Table 3. Mean, minimum (Min), maximum (Max), coefficient of variation (Coeff.var.) and number of samples (N) of variables measured during 2009 high water field mission.

\begin{tabular}{|c|c|c|c|c|c|}
\hline Variables & Mean & Min & Max & Coeff.Var. & $\mathrm{N}$ \\
\hline Transparency (m) & 1.58 & 0.60 & 2.80 & 33.24 & 136 \\
\hline Temperature $\left({ }^{\circ} \mathrm{C}\right)$ & 27.18 & 26.50 & 28.92 & 1.90 & 136 \\
\hline $\mathrm{pH}$ & 6.79 & 6.35 & 7.01 & 1.69 & 136 \\
\hline $\mathrm{EC}\left(\mu \mathrm{S} . \mathrm{cm}^{-1}\right)$ & 105.21 & 50.00 & 123.00 & 18.92 & 136 \\
\hline Turbidity (NTU) & 8.74 & 0.50 & 31.30 & 76.88 & 136 \\
\hline $\mathrm{DO}\left(\mathrm{mg} \cdot \mathrm{L}^{-1}\right)$ & 2.36 & 0.86 & 6.98 & 33.13 & 136 \\
\hline $\operatorname{SIM}\left(m g \cdot L^{-1}\right)$ & 0.92 & 0.05 & 3.02 & 87.21 & 41 \\
\hline $\operatorname{SOM}\left(\mathrm{mg}^{\mathrm{L}} \mathrm{L}^{-1}\right)$ & 1.17 & 0.24 & 2.00 & 37.61 & 41 \\
\hline Chlorophyll-a $\left(\mu \mathrm{g} \cdot \mathrm{L}^{-1}\right)$ & 0.29 & 0.11 & 0.81 & 45.89 & 41 \\
\hline Pheophityn $\left(\mu \mathrm{g} . \mathrm{L}^{-1}\right)$ & 0.24 & 0.00 & 0.64 & 66.48 & 41 \\
\hline Nitrogen $\left(\mathrm{mg} \mathrm{L}^{-1}\right)$ & 0.18 & 0.03 & 0.31 & 34.64 & 41 \\
\hline Phosphorus ( $\left.\mu \mathrm{g} \cdot \mathrm{L}^{-1}\right)$ & 105.11 & 50.68 & 179.42 & 29.68 & 41 \\
\hline $\mathrm{DIC}\left(\mathrm{mg} \cdot \mathrm{L}^{-1}\right)$ & 8.16 & 3.56 & 9.58 & 15.23 & 41 \\
\hline $\mathrm{DOC}\left(\mathrm{mg}^{\left.-\mathrm{L}^{-1}\right)}\right.$ & 4.62 & 2.02 & 6.31 & 20.87 & 41 \\
\hline
\end{tabular}

EC: Electrical conductivity; DO: dissolved oxygen; SIM: suspended inorganic matter; SOM: suspended organic matter; DIC: dissolved inorganic carbon; DOC: dissolved organic carbon

Table 4. Mean, minimum (Min), maximum (Max), coefficient of variation (Coeff.var.) and number of samples (N) of variables measured during 2009 low water field mission.

\begin{tabular}{|c|c|c|c|c|c|}
\hline Variables & Mean & Min & Max & Coeff.Var. & $\mathrm{N}$ \\
\hline Transparency (m) & 0.32 & 0.05 & 1.00 & 66.53 & 96 \\
\hline Temperature $\left({ }^{\circ} \mathrm{C}\right)$ & 31.22 & 27.98 & 33.91 & 3.80 & 96 \\
\hline $\mathrm{pH}$ & 7.28 & 6.21 & 8.79 & 6.42 & 96 \\
\hline $\mathrm{EC}\left(\mu \mathrm{S} . \mathrm{cm}^{-1}\right)$ & 174.42 & 54.00 & 378.00 & 59.11 & 96 \\
\hline Turbidity (NTU) & 60.42 & 2.70 & 565.00 & 135.04 & 96 \\
\hline $\mathrm{DO}\left(\mathrm{mg} \cdot \mathrm{L}^{-1}\right)$ & 5.65 & 0.72 & 14.02 & 49.31 & 96 \\
\hline $\operatorname{SIM}\left(m g \cdot L^{-1}\right)$ & 57.68 & 1.52 & 590.39 & 169.49 & 75 \\
\hline $\mathrm{SOM}\left(\mathrm{mg} \cdot \mathrm{L}^{-1}\right)$ & 21.14 & 4.47 & 202.96 & 121.59 & 75 \\
\hline Chlorophyll-a $\left(\mu \mathrm{g} \cdot \mathrm{L}^{-1}\right)$ & 113.03 & 2.90 & $2,061.29^{*}$ & 211.06 & 75 \\
\hline Pheophityn $\left(\mu \mathrm{g} \cdot \mathrm{L}^{-1}\right)$ & 18.38 & 1.63 & 73.32 & 72.32 & 75 \\
\hline Nitrogen (mg. $\mathrm{L}^{-1}$ ) & 1.62 & 0.04 & 10.31 & 83.35 & 75 \\
\hline Phosphorus $\left(\mu \mathrm{g} . \mathrm{L}^{-1}\right)$ & 220.99 & 38.05 & $1,094.89$ & 95.00 & 75 \\
\hline $\mathrm{DIC}\left(\mathrm{mg} \cdot \mathrm{L}^{-1}\right)$ & 16.06 & 0.49 & 40.04 & 69.64 & 75 \\
\hline $\mathrm{DOC}\left(\mathrm{mg} \cdot \mathrm{L}^{-1}\right)$ & 11.47 & 1.71 & 23.39 & 53.87 & 75 \\
\hline
\end{tabular}

*Phytoplankton bloom. EC: Electrical conductivity; DO: dissolved oxygen; SIM: suspended inorganic matter; SOM: suspended organic matter; DIC: dissolved inorganic carbon; DOC: dissolved organic carbon. 
Table 5. Mean, minimum (Min), maximum (Max), coefficient of variation (Coeff.var.) and number of samples (N) of variables measured during 2010 low water field mission.

\begin{tabular}{|c|c|c|c|c|c|}
\hline Variables & Mean & Min & Max & Coeff.Var. & $\mathrm{N}$ \\
\hline Transparency (m) & 0.44 & 0.10 & 1.00 & 55.22 & 64 \\
\hline Temperature $\left({ }^{\circ} \mathrm{C}\right)$ & 31.33 & 28.80 & 34.70 & 4.26 & 64 \\
\hline $\mathrm{pH}$ & 7.06 & 6.46 & 7.62 & 3.61 & 64 \\
\hline $\mathrm{EC}\left(\mu \mathrm{S} . \mathrm{cm}^{-1}\right)$ & 151.25 & 35.00 & 313.00 & 56.79 & 64 \\
\hline Turbidity (NTU) & 96.46 & 6.00 & 527.20 & 95.17 & 64 \\
\hline $\mathrm{DO}\left(\mathrm{mg} \cdot \mathrm{L}^{-1}\right)$ & 5.24 & 1.86 & 9.70 & 25.86 & 64 \\
\hline $\operatorname{SIM}\left(m g \cdot L^{-1}\right)$ & 27.15 & 1.60 & 122.19 & 106.58 & 45 \\
\hline $\operatorname{SOM}\left(m g \cdot L^{-1}\right)$ & 10.69 & 2.31 & 27.86 & 47.92 & 45 \\
\hline Chlorophyll-a $\left(\mu \mathrm{g} \cdot \mathrm{L}^{-1}\right)$ & 148.07 & 1.13 & $2,589.23^{*}$ & 330.02 & 45 \\
\hline Pheophityn ( $\left.\mu \mathrm{g} . \mathrm{L}^{-1}\right)$ & 15.67 & 1.35 & 95.59 & 87.35 & 45 \\
\hline Nitrogen (mg.L-1) & 1.17 & 0.17 & 4.07 & 72.39 & 45 \\
\hline Phosphorus ( $\left.\mu g . L^{-1}\right)$ & 210.78 & 59.68 & 701.81 & 67.65 & 45 \\
\hline DIC (mg.L-1) & 15.19 & 3.58 & 31.60 & 63.11 & 45 \\
\hline $\mathrm{DOC}\left(\mathrm{mg} \cdot \mathrm{L}^{-1}\right)$ & 8.44 & 1.85 & 22.55 & 57.89 & 45 \\
\hline
\end{tabular}

*Phytoplankton bloom. EC: Electrical conductivity; DO: dissolved oxygen; SIM: suspended inorganic matter; SOM: suspended organic matter; DIC: dissolved inorganic carbon; DOC: dissolved organic carbon.

Table 6. Two-sample Kolmogorov-Smirnov test (Dmax) between hydrograph phases and main rivers for temperature, water transparency and electrical conductivity and inorganic and organic dissolved carbon. Sig $1 \%$ indicates the variables that display different distributions.

\begin{tabular}{|c|c|c|c|c|c|c|}
\hline Parameter & Hydrograph phase & Water body & Year comparison & Dmax & Dcrit. & Sig. $1 \%$ \\
\hline \multirow{11}{*}{ Temperature } & \multirow[t]{2}{*}{ High } & \multirow[t]{2}{*}{ RM $x$ LM } & 2009 & 0.91 & 0.45 & Yes \\
\hline & & & $2009 \times 2008$ & 1.00 & 0.27 & Yes \\
\hline & \multirow[t]{3}{*}{ High $x$ low } & \multirow[t]{3}{*}{ RM } & $2009 \times 2009$ & 0.96 & 0.23 & Yes \\
\hline & & & $2009 \times 2010$ & 0.99 & 0.27 & Yes \\
\hline & & & $2008 \times 2009$ & 0.21 & 0.29 & - \\
\hline & & RM & $2008 \times 2010$ & 0.23 & 0.32 & - \\
\hline & & & $2009 \times 2010$ & 0.12 & 0.28 & - \\
\hline & \multirow[t]{4}{*}{ Low } & \multirow{2}{*}{ Japurá x Solimões } & 2009 & 0.83 & 0.91 & - \\
\hline & & & 2010 & 0.80 & 1.03 & - \\
\hline & & Japurá & $2009 \times 2010$ & 1.00 & 0.95 & Yes \\
\hline & & Solimões & $2009 \times 2010$ & 0.80 & 0.99 & - \\
\hline \multirow{11}{*}{ Water transparency } & \multirow[t]{2}{*}{ High } & \multirow[t]{2}{*}{ RM $x$ LM } & 2009 & 0.95 & 0.49 & Yes \\
\hline & & & $2009 \times 2008$ & 0.95 & 0.48 & Yes \\
\hline & \multirow[t]{3}{*}{ High x low } & \multirow[t]{3}{*}{ RM } & $2009 \times 2009$ & 0.95 & 0.33 & Yes \\
\hline & & & $2009 \times 2010$ & 0.93 & 0.38 & Yes \\
\hline & & & $2008 \times 2009$ & 0.31 & 0.45 & - \\
\hline & & RM & $2008 \times 2010$ & 0.24 & 0.49 & - \\
\hline & & & $2009 \times 2010$ & 0.42 & 0.34 & Yes \\
\hline & \multirow[t]{4}{*}{ Low } & \multirow{2}{*}{ Japurá x Solimões } & 2009 & 0.80 & 1.09 & - \\
\hline & & & 2010 & 0.80 & 1.03 & - \\
\hline & & Japurá & $2009 \times 2010$ & 0.60 & 1.03 & - \\
\hline & & Solimões & $2009 \times 2010$ & 1.00 & 1.09 & - \\
\hline \multirow{11}{*}{ Electrical conductivity } & \multirow[t]{2}{*}{ High } & \multirow[t]{2}{*}{ RM $x$ LM } & 2009 & 1.00 & 0.45 & Yes \\
\hline & & & $2009 \times 2008$ & 0.72 & 0.27 & Yes \\
\hline & \multirow[t]{5}{*}{ High $x$ low } & \multirow[t]{3}{*}{ RM } & $2009 \times 2009$ & 0.55 & 0.23 & Yes \\
\hline & & & $2009 \times 2010$ & 0.44 & 0.27 & Yes \\
\hline & & & $2008 \times 2009$ & 0.37 & 0.29 & Yes \\
\hline & & RM & $2008 \times 2010$ & 0.32 & 0.32 & Yes \\
\hline & & & $2009 \times 2010$ & 0.18 & 0.28 & - \\
\hline & \multirow[t]{4}{*}{ Low } & \multirow{2}{*}{ Japurá x Solimões } & 2009 & 0.83 & 0.91 & - \\
\hline & & & 2010 & 0.80 & 1.03 & - \\
\hline & & Japurá & $2009 \times 2010$ & 1.00 & 0.95 & Yes \\
\hline & & Solimões & $2009 \times 2010$ & 0.80 & 1.03 & - \\
\hline
\end{tabular}

Rm: lakes of the right margin of Japurá lakes, LM: Left margin of Japurá River. High: high water phase (flood), Low: low water phase (drought). 
Table 7. Two-sample Kolmogorov-Smirnov test (Dmax) between hydrograph phases and main rivers for $\mathrm{pH}$, turbidity and dissolved oxygen and inorganic and organic dissolved carbon. Sig 1\% indicates the variables that display different distributions.

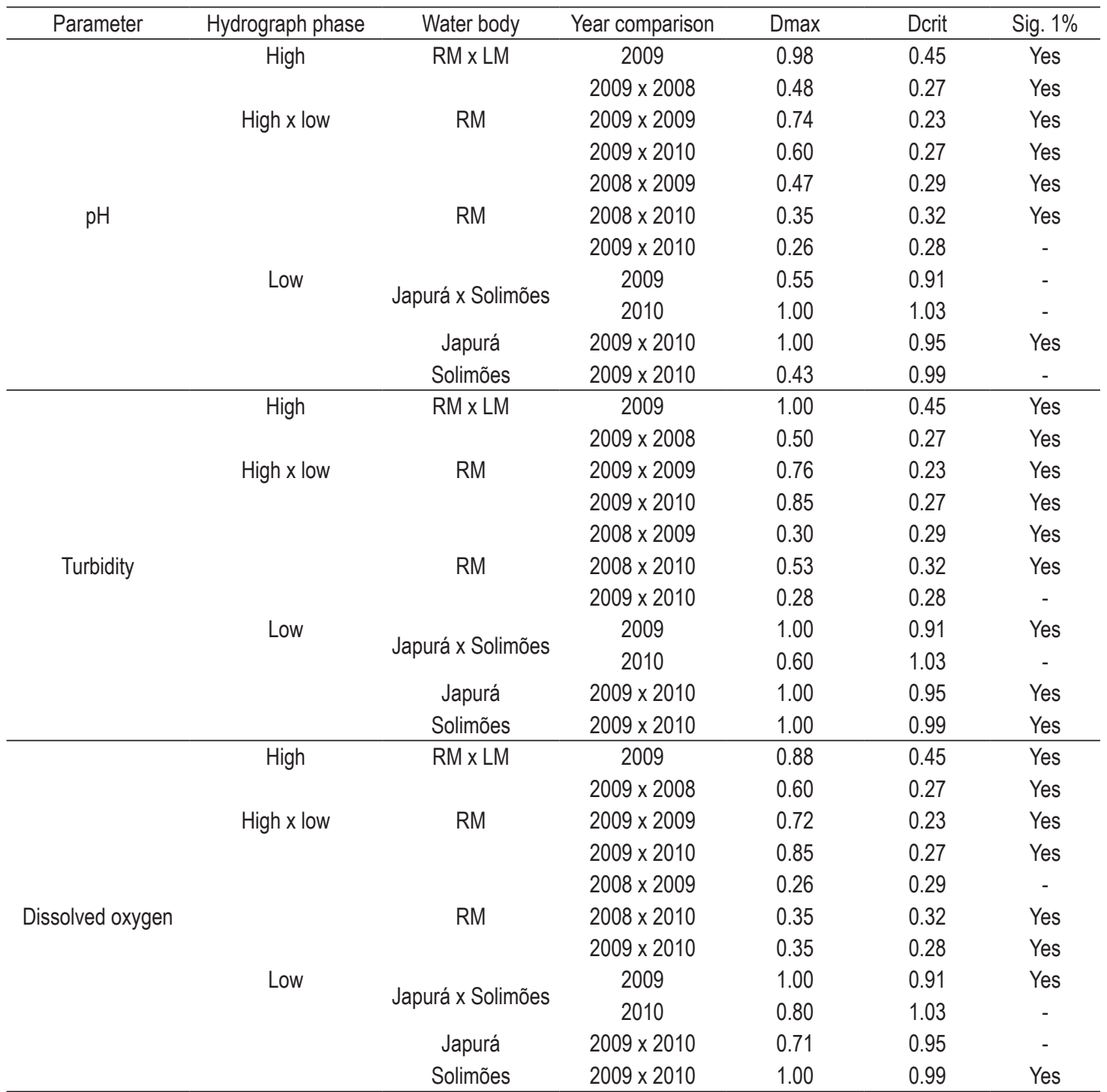

Rm: lakes of the right margin of Japurá lakes, LM: Left margin of Japurá River. High: high water phase (flood), Low: low water phase (drought).

On the other hand, $\mathrm{pH}$ mean values were similar between hydrograph phases, but minimum and maximum values ranged from acid to basic waters. During HWP, the $\mathrm{pH}$ ranged from 6.35 to 7.01 , with a mean of 6.79. Right and left margin lakes presented acid waters with mean values of 6.82 and 6.56, respectively. During LWP, $\mathrm{pH}$ values varied from 6.21 (2009) to 8.79 (2009), with mean values ranging from 6.99 (2008) to 7.28 (2009). Solimóes presented the highest mean values in 2009 (7.39) and 2010 (7.32) drought events; however the maximum value was registered in right margin lakes in 2009 drought (8.79) (Tables 2 to 5).
Electrical conductivity ranged from 50 to $123 \mu \mathrm{S} . \mathrm{cm}^{-1}$, with mean values of $105.21 \mu \mathrm{S} . \mathrm{cm}^{-1}$, in HWP. During LWP the condutivity varied from $35 \mu \mathrm{S} . \mathrm{cm}^{-1}$ (2010) to $378 \mu \mathrm{S} . \mathrm{cm}^{-1}$ (2009), with mean values ranging from $151.25 \mu$ S. $\mathrm{cm}^{-1}$ (2010) to $174.42 \mu \mathrm{S} . \mathrm{cm}^{-1}$ (2009). Conductivity of the right margin lakes was two times higher than that of the left margin in HWP. During LWP, Japurá had the lowest mean values, below $75 \mu$ S.cm ${ }^{-1}$ (2009) while right margin lakes and Solimóes River had the highest values, above $162.81 \mu \mathrm{S} . \mathrm{cm}^{-1}$ (2010) and $100.83 \mu{\mathrm{S} . \mathrm{cm}^{-1}}^{-12009)}$ ), respectively (Tables 2 to 5 ).

Turbidity ranged from 0.5 NTU to 31.30 NTU, with a mean of 8.74 NTU in HWP, and from 3.92 
Table 8. Two-sample Kolmogorov-Smirnov test (Dmax) between hydrograph phases and main rivers for total suspended matter, suspended inorganic and inorganic matter, chlorophyll- $a$ and pheophytin and inorganic and organic dissolved carbon. Sig 1\% indicates the variables that display different distributions.

\begin{tabular}{|c|c|c|c|c|c|c|}
\hline Parameter & Hydrograph phase & Water body & Year comparison & Dmax & Dcrit & Sig. $1 \%$ \\
\hline \multirow{7}{*}{$\begin{array}{l}\text { Total suspended } \\
\text { matter }\end{array}$} & \multirow{2}{*}{ High x low } & \multirow{2}{*}{ RM } & $2009 \times 2009$ & 1.00 & 0.32 & Yes \\
\hline & & & $2009 \times 2010$ & 1.00 & 0.38 & Yes \\
\hline & \multirow{5}{*}{ Low } & RM & $2009 \times 2010$ & 0.32 & 0.34 & - \\
\hline & & Japurá x & 2009 & 0.75 & 1.09 & - \\
\hline & & Solimões & 2010 & 0.60 & 1.03 & - \\
\hline & & Japurá & $2009 \times 2010$ & 0.80 & 1.03 & - \\
\hline & & Solimões & $2009 \times 2010$ & 0.60 & 1.09 & - \\
\hline \multirow{7}{*}{$\begin{array}{c}\text { Suspended inorganic } \\
\text { matter }\end{array}$} & \multirow{2}{*}{ High x low } & \multirow{2}{*}{ RM } & $2009 \times 2009$ & 0.94 & 0.32 & Yes \\
\hline & & & $2009 \times 2010$ & 0.87 & 0.38 & Yes \\
\hline & \multirow{5}{*}{ Low } & RM & $2009 \times 2010$ & 0.32 & 0.34 & - \\
\hline & & Japurá $x$ & 2009 & 0.75 & 1.09 & - \\
\hline & & Solimões & 2010 & 0.60 & 1.03 & - \\
\hline & & Japurá & $2009 \times 2010$ & 0.80 & 1.03 & - \\
\hline & & Solimões & $2009 \times 2010$ & 0.60 & 1.09 & - \\
\hline \multirow{7}{*}{$\begin{array}{l}\text { Suspended organic } \\
\text { matter }\end{array}$} & \multirow{2}{*}{ High x low } & \multirow{2}{*}{ RM } & $2009 \times 2009$ & 1.00 & 0.32 & Yes \\
\hline & & & $2009 \times 2010$ & 1.00 & 0.38 & Yes \\
\hline & \multirow{5}{*}{ Low } & RM & $2009 \times 2010$ & 0.31 & 0.34 & - \\
\hline & & Japurá $x$ & 2009 & 0.80 & 1.09 & - \\
\hline & & Solimões & 2010 & 0.80 & 1.03 & - \\
\hline & & Japurá & $2009 \times 2010$ & 0.40 & 1.03 & - \\
\hline & & Solimões & $2009 \times 2010$ & 1.00 & 1.09 & - \\
\hline \multirow{7}{*}{ Chlorophyll-a } & \multirow{2}{*}{ High $x$ low } & \multirow{2}{*}{ RM } & $2009 \times 2009$ & 1.00 & 0.32 & Yes \\
\hline & & & $2009 \times 2010$ & 1.00 & 0.38 & Yes \\
\hline & \multirow{5}{*}{ Low } & $\mathrm{RM}$ & $2009 \times 2010$ & 0.35 & 0.34 & Yes \\
\hline & & Japurá x & 2009 & 0.75 & 1.09 & - \\
\hline & & Solimões & 2010 & 1.00 & 1.03 & - \\
\hline & & Japurá & $2009 \times 2010$ & 0.80 & 1.03 & - \\
\hline & & Solimões & $2009 \times 2010$ & 0.40 & 1.09 & - \\
\hline \multirow{7}{*}{ Pheophytin } & \multirow{2}{*}{ High $\times$ low } & \multirow{2}{*}{ RM } & $2009 \times 2009$ & 1.00 & 0.32 & Yes \\
\hline & & & $2009 \times 2010$ & 1.00 & 0.38 & Yes \\
\hline & \multirow{5}{*}{ Low } & $\mathrm{RM}$ & $2009 \times 2010$ & 0.48 & 0.34 & Yes \\
\hline & & Japurá $x$ & 2009 & 1.00 & 1.09 & - \\
\hline & & Solimões & 2010 & 1.00 & 1.03 & - \\
\hline & & Japurá & $2009 \times 2010$ & 0.80 & 1.03 & - \\
\hline & & Solimões & $2009 \times 2010$ & 1.00 & 1.09 & - \\
\hline
\end{tabular}

Rm: lakes of the right margin of Japurá lakes, High: high water phase (flood), Low: low water phase (drought).

NTU to 565 NTU in LWP. Mean values during drought events ranged from 23.82 NTU, in right margin lakes in 2008, to 218.42 NTU in Solimóes River in 2010. Left margin lakes were four times more turbid than right margin lakes, during HWP. Solimões and Japurá Rivers were more turbid than right margin lakes during LWP (Tables 2 to 5).

Dissolved oxygen mean concentration varied from $4.23 \mathrm{mg} . \mathrm{L}^{-1}$ (2008) to $5.65 \mathrm{mg} . \mathrm{L}^{-1}$ (2009) in LWP. In 2009 high water phase, it presented the lowest mean value $\left(2.36 \mathrm{mg} . \mathrm{L}^{-1}\right)$. During HWP mean oxygen concentrations were higher in left margin lakes $\left(3.33 \mathrm{mg} \cdot \mathrm{L}^{-1}\right)$ than in right margin lakes (2.24 mg. $\left.\mathrm{L}^{-1}\right)$. During 2009 drought event, dissolved oxygen was higher in right margin lakes than in main rivers. However in 2010 Solimóes and Japurá had more oxygenated waters (Tables 2 to 5).

Suspended inorganic matter (SIM) varied from 0.05 to $3.02 \mathrm{mg} . \mathrm{L}^{-1}$ with a mean value of $0.92 \mathrm{mg} . \mathrm{L}^{-1}$, during HWP. During LWP inorganic fraction varied from 1.52 to $590.39 \mathrm{mg} . \mathrm{L}^{-1}$, with mean values of $57.68 \mathrm{mg} \cdot \mathrm{L}^{-1}(2009)$ and $27.15 \mathrm{mg} . \mathrm{L}^{-1}$ (2010). The maximum concentration was found in right margin lakes $\left(590.39\right.$ mg. $\left.\mathrm{L}^{-1}\right)$ 
Table 9. Two-sample Kolmogorov-Smirnov test (Dmax) between hydrograph phases and main rivers for nitrogen, phosphorus, total dissolved carbon and inorganic and organic dissolved carbon. Sig 1\% indicates the variables that display different distributions.

\begin{tabular}{|c|c|c|c|c|c|c|}
\hline Parameter & Hydrograph phase & Water body & Year comparison & Dmax & Dcrit & Sig. $1 \%$ \\
\hline \multirow{7}{*}{ Nitrogen } & \multirow{2}{*}{ High $\times$ low } & \multirow{2}{*}{ RM } & $2009 \times 2009$ & 0.97 & 0.32 & Yes \\
\hline & & & $2009 \times 2010$ & 0.98 & 0.38 & Yes \\
\hline & \multirow{5}{*}{ Low } & $\mathrm{RM}$ & $2009 \times 2010$ & 0.47 & 0.34 & Yes \\
\hline & & \multirow{2}{*}{ Japurá x Solimões } & 2009 & 0.55 & 1.09 & - \\
\hline & & & 2010 & 0.40 & 1.03 & - \\
\hline & & Japurá & $2009 \times 2010$ & 0.80 & 1.03 & - \\
\hline & & Solimões & $2009 \times 2010$ & 0.80 & 1.09 & - \\
\hline \multirow{7}{*}{ Phosphurus } & \multirow{3}{*}{ High x low } & \multirow{2}{*}{ RM } & $2009 \times 2009$ & 0.56 & 0.32 & Yes \\
\hline & & & $2009 \times 2010$ & 0.70 & 0.38 & Yes \\
\hline & & RM & $2009 \times 2010$ & 0.47 & 0.34 & Yes \\
\hline & \multirow{4}{*}{ Low } & \multirow{2}{*}{ Japurá x Solimões } & 2009 & 0.35 & 1.24 & - \\
\hline & & & 2010 & 0.60 & 1.15 & - \\
\hline & & Japurá & $2009 \times 2010$ & 1.00 & 1.15 & - \\
\hline & & Solimões & $2009 \times 2010$ & 0.75 & 1.24 & - \\
\hline \multirow{7}{*}{ Total dissolved carbon } & \multirow{3}{*}{ High $x$ low } & \multirow{2}{*}{ RM } & $2009 \times 2009$ & 0.67 & 0.32 & Yes \\
\hline & & & $2009 \times 2010$ & 0.91 & 0.38 & Yes \\
\hline & & RM & $2009 \times 2010$ & 0.47 & 0.34 & Yes \\
\hline & \multirow{4}{*}{ Low } & \multirow{2}{*}{ Japurá x Solimões } & 2009 & 0.80 & 1.09 & - \\
\hline & & & 2010 & 0.80 & 1.03 & - \\
\hline & & Japurá & $2009 \times 2010$ & 0.60 & 1.03 & - \\
\hline & & Solimões & $2009 \times 2010$ & 0.60 & 1.09 & - \\
\hline \multirow{7}{*}{ Dissolved inorganic carbon } & \multirow{3}{*}{ High $x$ low } & \multirow{2}{*}{$\mathrm{RM}$} & $2009 \times 2009$ & 0.62 & 0.32 & Yes \\
\hline & & & $2009 \times 2010$ & 0.88 & 0.38 & Yes \\
\hline & & RM & $2009 \times 2010$ & 0.47 & 0.34 & Yes \\
\hline & \multirow{4}{*}{ Low } & \multirow{2}{*}{ Japurá x Solimões } & 2009 & 0.80 & 1.09 & - \\
\hline & & & 2010 & 0.80 & 1.03 & - \\
\hline & & Japurá & $2009 \times 2010$ & 0.80 & 1.03 & - \\
\hline & & Solimões & $2009 \times 2010$ & 0.80 & 1.09 & - \\
\hline \multirow{7}{*}{ Dissolved organic carbon } & \multirow{3}{*}{ High $\times$ low } & \multirow{2}{*}{ RM } & $2009 \times 2009$ & 0.80 & 0.32 & Yes \\
\hline & & & $2009 \times 2010$ & 0.93 & 0.38 & Yes \\
\hline & & RM & $2009 \times 2010$ & 0.47 & 0.34 & Yes \\
\hline & \multirow{4}{*}{ Low } & \multirow{2}{*}{ Japurá x Solimões } & 2009 & 0.75 & 1.09 & - \\
\hline & & & 2010 & 0.60 & 1.03 & - \\
\hline & & Japurá & $2009 \times 2010$ & 0.40 & 1.03 & - \\
\hline & & Solimões & $2009 \times 2010$ & 0.75 & 0.98 & - \\
\hline
\end{tabular}

Rm: lakes of the right margin of Japurá lakes, High: high water phase (flood), Low: low water phase (drought).

during LWP. Solimões and Japurá rivers mean concentrations were higher than that of the right margin lakes, and Solimóes River presented the highest mean concentration (134.6 mg.L $\mathrm{L}^{-1}$ ) which was more than two times the right margin lakes SIM mean concentration (51.1 mg. $\mathrm{L}^{-1}$ ) (Tables 2 to 5).

Suspended organic matter (SOM) varied from 0.24 to $2 \mathrm{mg} . \mathrm{L}^{-1}$, with a mean value of $1.17 \mathrm{mg} . \mathrm{L}^{-1}$ during HWP. During drought events SOM varied from 2.31 to $202.96 \mathrm{mg} . \mathrm{L}^{-1}$, with mean concentrations of $21.14 \mathrm{mg} \cdot \mathrm{L}^{-1}(2009)$ and 10.69 mg.L $L^{-1}$ (2010). Right margin lakes presented the highest mean concentration in both drought events, with $22.2 \mathrm{mg} . \mathrm{L}^{-1}(2009)$ and $11.2 \mathrm{mg} . \mathrm{L}^{-1}$ (2010). Japurá had lower mean concentration in 2009 (9.9 mg. $\left.\mathrm{L}^{-1}\right)$ and higher in 2010 (10.7 mg. $\left.\mathrm{L}^{-1}\right)$ compared to Solimóes River in the same period (17.2 mg.L. $\mathrm{L}^{-1}$ in 2009 and $7.4 \mathrm{mg} . \mathrm{L}^{-1}$ in 2010) (Tables 2 to 5 ).

During HWP, chlorophyll- $a$ and pheophytin were lower than $1 \mu \mathrm{g} . \mathrm{L}^{-1}$ in all samples. Nonetheless during LWP chlorophyll-a mean concentration was three hundred times higher in 2010 LWP $\left(148 \mu \mathrm{g} . \mathrm{L}^{-1}\right)$ than in 2009 HWP, and in 2009 drought the mean chlorophyll-a concentration was $113.03 \mu \mathrm{g} . \mathrm{L}^{-1}$. Solimôes and Japurá mean 
concentration during LWP was lower than $10.2 \mu \mathrm{g} . \mathrm{L}^{-1}$ while right margin lakes mean concentration was higher than $127.3 \mu \mathrm{g} \cdot \mathrm{L}^{-1}$. Maximum chlorophyll concentration occurred in right margin lakes in 2010 drought with 2589.23 $\mu \mathrm{g} . \mathrm{L}^{-1}$, where there was a phytoplankton bloom. Pheophytin varied from $1.35 \mu \mathrm{g} . \mathrm{L}^{-1}$ to $95.59 \mu \mathrm{g} . \mathrm{L}^{-1}$ during LWP. Mean concentration in HWP was 0.2 $\mu \mathrm{g} . \mathrm{L}^{-1}$. In LWP, mean concentrations were 18.38

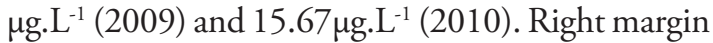
lakes had the highest mean $\left(20.1 \mu \mathrm{g} \cdot \mathrm{L}^{-1}\right.$ in 2009) and Solimóes river the lowest one $\left(2.6 \mu \mathrm{g} . \mathrm{L}^{-1}\right.$ in 2009) during LWP (Tables 2 to 5).

Mean phosphorus concentration was $105.11 \mu \mathrm{g} . \mathrm{L}^{-1}$, during HWP, varying from $50.68 \mu \mathrm{g} . \mathrm{L}^{-1}$ to $179.42 \mu \mathrm{g} . \mathrm{L}^{-1}$. During LWP total phosphorus mean concentrations were two times higher (220.99 $\mu \mathrm{g} . \mathrm{L}^{-1}$ in 2009 and $210.78 \mu \mathrm{g} . \mathrm{L}^{-1}$ in 2010) than in flood period, with a maximum concentration of $1094.89 \mu \mathrm{g} . \mathrm{L}^{-1}$ in 2009. Right margin lakes had highest mean values during HWP and LWP than the main rivers in 2009 LWP. However during 2010 LWP, Solimôes River presented the highest mean value with $233.8 \mu \mathrm{g} . \mathrm{L}^{-1}$ (Tables 2 to 5).

Nitrogen concentration was lower than 0.32 mg. $\mathrm{L}^{-1}$ in HWP, with a mean value of $0.18 \mathrm{mg} . \mathrm{L}^{-1}$. During LWP, nitrogen varied from $0.04 \mathrm{mg} . \mathrm{L}^{-1}$ to $10.31 \mathrm{mg} . \mathrm{L}^{-1}$, with mean values of $1.62 \mathrm{mg} . \mathrm{L}^{-1}$ (2009) and 1.17 mg.L $\mathrm{L}^{-1}$ (2010). Right margin lakes had the highest mean values during LWP. In 2009, those lakes had six times more nitrogen than Japurá and 4 times than Solimóes mean values. In 2010, both Japura and Solimóes had almost 5 times less nitrogen than the lakes located in the right margin of Japurá River (Tables 2 to 5).

Dissolved inorganic carbon was higher during droughts with mean concentrations of $16.06 \mathrm{mg} . \mathrm{L}^{-1}$ (2009) and 15.19 mg.L ${ }^{-1}$ (2010). In HWP mean concentration was $8.16 \mathrm{mg} . \mathrm{L}^{-1}$ with a maximum value of $9.58 \mathrm{mg} . \mathrm{L}^{-1}$. The highest inorganic carbon mean concentration was observed in right margin lakes and in Solimóes River in both years. Japurá River values during LWP were even lower than the concentrations found during HWP in right margin lakes (Tables 2 to 5).

The organic fraction was also higher during LWP with mean concentrations of $11.47 \mathrm{mg} . \mathrm{L}^{-1}$ (2009) and $8.44 \mathrm{mg} . \mathrm{L}^{-1}$ (2010), and during HWP the mean value was $4.62 \mathrm{mg} . \mathrm{L}^{-1}$. The lakes located on the right margin presented the highest values. Solimóes and Japurá River concentrations during the LWP were lower than the organic carbon concentration of the right margin lakes during HWP (Tables 2 to 5).

\section{Discussion}

The results showed that the studied water bodies have high variability on all measured chemical and physical variables between hydrograph phases, among main rivers and between opposite margins of Japurá River.The limnological variability among hydrological phases, and also between lakes on opposite margins and between main rivers points to the influence of several factors, such as, water volume, main river input, lake morphometry and connection to the main channel/river.

The seasonal flood homogenizes the water masses, all the lakes are interconnected with the main channels and rivers, receiving a huge volume of water, and thus promoting the lowest amplitudes for all variables were observed during high water phase. During the drought the lakes are isolated with poor or no connection to the main channels and rivers and can be considered as single units or actual lentic systems, influenced by their local immediate environment. This condition may explain the fact that they displayed the highest values and amplitudes of all variables during the low water period. This pattern was already observed elsewhere in the Amazon (Carvalho et al., 2001; Abdo and Silva, 2004; Almeida and Melo, 2009), and also in Mamirauá lakes, where Henderson (1999) and Queiroz (2007) noted similar patterns for conductivity, temperature, dissolved oxygen and water transparency, with lower values during flood and higher during drought (except for water transparency). Water volume in lakes and channels, during dry season, is much lower than in other seasons, thus many of them might remain isolated from the main channel and more turbid. Therefore the surface irradiance increases the water column temperature (Alcântara, 2010). However, during the rainy season, the large volume of flowing water in the floodplain turns the lakes into almost lotic system since they gain direction and flow, and thus, smaller residence time, increased turbulence and changes in water-air interface, which also contributes to lower the temperature. Besides that, the water column is more transparent, which favors light penetration and, consequently, the energy dissipation within a higher water volume. This enlarged water volume in the high water also explains the lower temperatures in this season. In addition, the flood period of this region is characterized by low solar radiation, increased cloud cover and high precipitation, 
opposite to the dry season (Ceballos et al., 2004; CPTEC, 2009; NASA, 2009).

During low water season, the lakes and channels are shallower and are primarily fed by channels bearing inorganic matter from main rivers. Furthermore, they are more affected by wind (because they are shallow), causing sediment resuspension. Nevertheless, during high water phase the lakes are fed by a huge water volume which comes in a diffuse way to the lakes throughout the forest and channels. Particulate material is deposited along the way, and when the water reaches the lakes there is almost no suspended matter and with probable low turbidity.

Similar to other variables, electrical conductivity was higher during droughts because during that period the lakes are isolated from main channels, with less water volume (shallower, higher solar incidence, and consequently, higher temperature and evaporation) with higher residence time, and consequently an intense ion exchange with the substrate. Inversely during high water phase those values are lower than that of the low water phase.

Barbosa et al. (2010) showed that Curuaí floodplain, Pará State (Lower Amazon) has higher magnitudes of $\mathrm{pH}$, turbidity, chlorophyll- $a$ and dissolved organic carbon during high water phase, than in Mamirauá floodplain. During HWP, Curuaí water was alcaline, more turbid, and with higher chlorophyll and organic carbon concentrations than in Mamirauá at the same period. However during LWP, this situation was inverse, with exception of turbidity when Curuai reached extreme mean values of 769 NTU while in Mamirauá mean turbidity were 23.82 (2008), 60.46 NTU (2009) and 96 NTU (in 2010). Mean dissolved inorganic carbon was almost two times higher in Mamirauá and chlorophyll- $a$ mean concentration was three times higher in Mamirauá than in Curuai during LWP. Water characteristics are dependent on local climate, soil, surrounding vegetation, human influence, and exhibited temporal and spatial variations due to internal and external processes (Maybec and Helmer, 1992; Wetzel, 2001). Curuai floodplain is located in eastern Amazon, $510 \mathrm{~km}$ distant from Manaus, in Pará State. There are 20,000 rural inhabitants living in 96 communities within the floodplain. This region is formed by 4 municipalities and has a strong rancher tradition with 234,678 cattle heads and 373,608 inhabitants (IBGE, 2006). According to Renó (2010), around $3,600 \mathrm{~km}^{2}$ of flooded forest were removed from the lower Amazon várzea between 1975-1981 and 2008, which represents $56 \%$ of that region.

On the other hand Mamirauá Sustainable Development Reserve, where human intervention is kept to minimum and deforestation is practically nonexistent. It is a highly conserved floodplain area where only 6,500 inhabitants are divided in 64 communities. The municipalities around the Reserve (6 in total) have up to 134,558 inhabitants and 8,677 cattle heads according to 2006 Census (IBGE, 2006).

Notwithstanding the limited (two hydrograph phases) and the large inter-annual variability of the hydrology forcing functions in those regions, it suggest that those differences between limnological conditions at Mamirauá and Curuai might be originated from anthropogenic activities and will in turn affect considerably those water properties and may pose a threat not only to human health but also for aquatic fauna and flora

This study showed the temporal (intra and interannual) and spatial (opposite margins and main rivers) variations that occurred in Mamirauá floodplain, and related them with the different phases of the hydrologic pulse. And this study also points out to the fact that less disturbed areas as Mamirauá Sustainable Development Reserve might be considered as future reference for comparison with more disturbed areas, and for modeling the effects of climate change and anthropogenic influences on Amazon aquatic ecosystem.

\section{Conclusions}

Limnological patterns of Amazon floodplain lakes are highly dependent on the flood pulse, and consequently on the surrounding environment, the main river input, and geographic location. Mamirauá floodplain lakes, an undisturbed Amazon region, can vary from an oligotrophic, with low primary productivity, during high water phase to a hyper eutrophic environment, high productivity, in low water phase. These changes, although highly contrasting shows how undisturbed regions respond to natural variance in such a diverse environment. The Amazon region is under anthropogenic pressure since the 70's and the consequences of these changes in the aquatic system is barely know. Thus these data will be fundamental for assessing not only human impact on aquatic systems but can also give support to future analyses on the effects of global climate changes in their biogeochemical cycles. 


\section{Acknowledgements}

The authors would like to thank the Fundação de Amparo à Pesquisa do Estado de São Paulo (FAPESP) Projects 08/07537-1 and 2008/066610 , to Conselho Nacional de Desenvolvimento Científico e Tecnológico (CNPq - Projects 477885/2007-1 and 550373/2010-1), and also to Mamirauá Sustainable Development Institute.

\section{References}

ABDO, MSA. and DA SILVA, CJ. 2004. Limnological characteristics of the water bodies of the Corutuba nesting site Brazil's Pantanal. Acta Limnol. Bras. vol. 16, no. 4, p. 359-368.

ALCÂNTARA, EH. 2010. Sensoriamento remoto da temperatura e dos fluxos de calor na superficie da água do reservatório de Itumbiara ( $G O$, Brasil). São José dos Campos: Instituto Nacional de Pesquisas Espaciais. [PhD thesis in Remote Sensing]

ALMEIDA, FF. and MELO, S. 2009. Limnological considerations about an Amazonian floodplain lake (Catalão lake - Amazonas State, Brazil). Acta Scientiarum. Biological Sciences, vol. 31, no. 4, p. 387-395.

ARRAUT, EM., MARMONTEL, M., MANTOVANI, JE., NOVO, EMLM., MACDONALD, DW. and KENWARD, RE. 2010. The lesser of two evils: seasonal migrations of Amazonian manatees in the Western Amazon. Journal of Zoology, vol. 180, no. 3, p. 247-256. http://dx.doi.org/10.1111/j.14697998.2009.00655.x

AUFDENKAMPE, AK., MAYORGA, E., HEDGES, JI., LLERENAC, C., QUAY, PD., GUDEMAN, J., KRUSCHE AV. and RICHEY, JE. 2007. Organic matter in the Peruvian headwaters of the Amazon: Compositional evolution from the Andes to the lowland Amazon mainstem. Organic Geochemistry, vol. 38, p. 337-364. http://dx.doi.org/10.1016/j. orggeochem.2006.06.003

AYRES, JMC. 1993. As Matas de Várzea do Mamirauá. Brasília: SCM-CNPq/MCT. 123 p. Série Estudos do Mamirauá, vol. 1.

BARBOSA, CCF., NOVO, EMLM., MELACK, JM., GASTIL-BUHL, M. and PEREIRA FILHO, W. 2010. Geospatial analysis of spatiotemporal patterns of $\mathrm{pH}$, total suspended sediment and chlorophyll-a on the Amazon floodplain. Limnology, vol. 11, p. 155-166. http://dx.doi.org/10.1007/s10201009-0305-5

CARVALHO, P., BINI, LM, THOMAZ, SM., OLIVEIRA, L., ROBERTSON, B., TAVECHIO, W. and DARWISCH, A. 2001. Comparative limnology of South American floodplain lakes and lagoons. Acta Scientiarum, vol. 23, no. 2, p. 265-273.

CHIPPS, SR., HUBBARD, DE., WELIN, KB., HARGERUD, NJ., POWELL, KA., THOMPSON, J. and JOHNSON, T. 2006. Association between wetland disturbance and biological attributes in floodplain wetlands. Wetlands, vol. 26, no. 2, p. 497-508.

CEBALlOS, JC., BOTTINO, MJ., SOUZA, JM. 2004. A simplified physical model for assessing solar radiation over Brazil using GOES-E imagery. J. Geoph. Res, vol. 109, D02211, p. 1-14.

Centro de Previsão de Tempo e Estudos Climáticos CPTEC. 2009. Monthly average of solar radiation. http://satelite.cptec.inpe.br/radiacao/?i=br\#/Glseries/ GLpentad0.htm.

Companhia de Pesquisa de Recursos Minerais - CPRM. 2009. Monitoramento Hidrológico. Relatórios de cheia, no. 23. Available from: <www.cprm.gov.br>.

EATON, AD., CLESCERI, L and GREENBERG, AE. 1995. Standard methods for the examination of water and wastewater. Washington: Am. Public Health Assoc.

FORSBERG, BR. DEVOL, AH., RICKEY, JE., MARTINELLI, LA., SANTOS, H. 1988. Factors controlling nutrient concentrations in Amazon floodplain lakes. Limnology and Oceanography, vol. 33, no. 1, p. 41-56.

FORSBERG, BR., ARAUJO-LIMA, CARM., MARTINELLI, LA., VICTORIA, RL. and BONASSI, JA. 1993. Autotrophic carbon sources for fish of the central Amazon. Ecology, vol. 74, p. 643-652.

GOLTERMAN, HL., CLYMO, H. and OHNSTAD, MA. 1978. Methods for physical and chemical analysis of freshwater. 2th ed. Oxford: Blackwell Scientific. 213p. JNP Handbook, no. 8.

HENDERSON, PA. O ambiente aquático da Reserva Mamirauá. In: QUEIROZ, H.L. \& CRAMPTON, W.G.R. (Eds.). Estratégias de Manejo de recursos Pesqueiros em Mamirauá. SCM, MCT-CNPq. Brasília, 1999. Cap. 1, p. 1-9.

Instituto Brasileiro de Geografia e Estatística - IBGE. 2006. Censo Agropecuário. <http://www.ibge.gov.br/ > Acesso em: 10 de outubro de 2009

JUNK, WJ. 1997. General aspects of floodplain ecology with special reference to Amazonian floodplains. In The Central Amazon Floodplain, Ecology of a Pulsing System (Ed. W.J. Junk), Springer-Verlag, Berlin, p 3-17.

JUNK, WJ. and DA SILVA, CJ. 1997. Mammals, reptiles and amphibians. In JUNK, WJ., ed. The Central Amazon Floodplain, Ecology of a Pulsing System. Berlin: Springer-Verlag. p. 409-417.

JUNK, WJ. OHLY, JJ., PIEDADE, MTF. and SOARES, MGM., 2000. The Central Amazon Floodplain Actual Use and Options for a Sustainable Management. Leiden: Backhuys Publishers. 584 p.

MCCLAIN,, ME. and NAIMAN, RJ. 2008. Andean influences on the biogeochemistry and ecology of the Amazon River. Bioscience, vol. 58, p. 325-338. http://dx.doi.org/10.1641/B580408 
MELACK, JM. and FISHER, TR. 1990. Comparative limnology of tropical floodplain lakes with an emphasis on the central Amazon. Acta Limnologica Brasiliensia, vol. 3, p. 1-48.

MELACK, JM. and FORSBERG, B. 2001. Biogeochemistry of Amazon floodplain lakes and associated wetlands. In MCCLAIN, ME., VICTORIA, RL. and RICHEY, JE., eds. The biogeochemistry of the Amazon basin and its role in a changing world. Oxford: Oxford Univ. Press. p. 235-276.

MEYBECK, M, and HELMER, R. 1992. An introduction to water quality. In: Chapman, D. Water quality assessment. Cambridge, University Press. 585 p.

MOREIRA-TURCQ, PF. SEYLER, P., GUYOT, JL. and ETCHEBER, H. 2003. Characteristics of organic matter in the mixing zone of the Rio Negro and Rio Solimóes of the Amazon River. Hydrological Processes, vol. 17, p. 1393- 1404.

National Aeronautics and Space Administration - NASA. 2009. Monthly $0.25^{\circ} \mathrm{x} 0.25^{\circ}$ TRMM (Tropical Rainfall Measuring Mission) http://trmm.gsfc.nasa. gov/, NASA Distrib. Active Arch. Cent., Goddard Space Flight Cent. Earth Sci., Greenbelt,Md.

NEILL, C., ELSENBEER, H., KRUSCHE, AV., LEHMANN, J., MARKEWITZ, D. and FIGUEIREDO, RO. 2006. Hydrological and Biogechemical Processes in a Changing Amazon: Results from small watershed studies and the Large-Scale Biosphere- Atmosphere Experiment. Hydrological Processes, vol. 20, p. 2467-2477. http:// dx.doi.org/10.1002/hyp.6210

NUSH, EA. 1980. Comparison of different methods for chlorophyll and phaeopigment determination. Archiv für Hydrobiologie, p. 14-39.

PAROLIN, P., SIMONE, O., HAASE, K.; WALDHOFF, D.; HOTTENBERGER, S.; KUHN, U.; KESSELMEIER, J.; KLEISS, B.; SCHIMIDT, W.; PIEDADE, MTF. and JUNK, WJ. 2004. Central Amazonian floodplain forests: Botanical Review. The New York Botanical Garden, vol. 70, no. 3, p. 357-380.

PIEDADE, MTF., LONG, SP. and JUNK, WJ. 1984. Leaf and canopy photosynthetic CO2 uptake of a stand of Echinochloa polystachya on the central Amazon floodplain. Oecology, vol. 97, p. 193-201.

QUEIROZ, HL. 2005. The Mamirauá sustainable development reserve. Estudos Avançados, vol. 19, no. 54, p. 183-203.

QUEIROZ, HL. 2007. Classification of water bodies based on biotic and abiotic parameters at the várzeas of Mamirauá Reserve, Central Amazon. Uakari, vol. 3, no. 2, p. 19-34.
RAMALHO, EE., MACEDO, J., VIEIRA, TM., VALSECCHI J., CALVIMONTES, J., MARMONTEL, M. and QUEIROZ, H. 2009. Hydrological cycle in a várzea environment at Mamirauá Sustainable Development Reserve Central Solimões, from 1990 to 2008. (Ciclo hidrológico nos ambientes de várzea da Reserva de desenvolvimento Susutentável Mamirauá- Médio Rio Solimóes, período de 1990 a 2008). Uakari, vol. 5, no. 1, p. 61-87.

RENÓ, VF. 2010. Avaliação do desflorestamento da várzea do Baixo Amazonas nas décadas de 1970 e 2000 a partir de imagens Landsat. Instituto Nacional de Pesquisas Espaciais, São José dos Campos, SP, Brasil. [Dissertação de Mestrado em Sensoriamento Remoto].

SANCHEZ-BOTERO JI. and ARAÚJO-LIMA, CARM. 2001. Aquatic macrophytes as fish nursery in Amazon River floodplain. (As macrófitas aquáticas como berçário para a ictiofauna da várzea do Rio Amazonas). Acta Amazonica, vol 31, no. 3, p. 437447.

SAINT-PAUL, U., ZUANON, J., CORREA, MV., GARCIA, M., FABRÉ, NN., BERGER, U. and JUNK, WJ. 2000. Fish communities in central Amazonian white- and blackwater floodplains. Environmental Biology of Fishes, vol. 57, p. 235-250. http://dx.doi.org/10.1023/a:1007699130333

SIEGEL, S. 1975. Nonparametric statistics for behavioral sciences. McGraw-Hill, Inc.

SIOLI, H. 1984. The Amazon: limnology and landscape ecology of a mighty tropical river and its basin. Dordrecht: Dr W. Junk Publishers.

SILVA, TS., COSTA, MPF. and MELACK, JM. 2010. A ssessment of two biomass estimation methods for aquatic vegetation growing on the Amazon floodplain. Aquatic Botany, vol. 92, p. 161-167. http://dx.doi.org/10.1016/j.aquabot.2009.10.015

TUNDISI, JG., TUNDISI, TM. and ROCHA, O. 2002. Ecossistema de águas interiores. In REBOUÇAS, A., BRAGA, B. and TUNDISI, JG., eds. Águas doces do Brasil: Capital ecológico, uso e conservação. São Paulo: Escrituras Editora. p. 161-202.

VALDERRAMA, GC. 1981. The simultaneous analysis of total nitrogen and total phosphorus in natural waters. Marine Chemistry, vol. 10, p. 109-122.

WETZEL, RG., 2001. Limnology: lake and river ecosystems. Elsevier Academic Press, San Diego, $1006 \mathrm{p}$.

WETZEL, RG. and LIKENS, GE. 1991. Limnological analysis. New York: Springer-Verlag. 391 p.

Received: 27 April 2011 Accepted: 21 September 2011 